\title{
EDITORIAL
}

\section{Obesity and asthma: location, location, location}

\author{
Stephanie A. Shore
}

$\mathbf{T}$ here is a worldwide epidemic of obesity. In the USA, the prevalence of obesity, defined as a body mass index $(\mathrm{BMI}) \geqslant 30 \mathrm{~kg} \cdot \mathrm{m}^{-2}$, has increased among adults aged 20 74 yrs from $\sim 15 \%$ in the late 1970 s to $\sim 35 \%$ in 2010 . Another third of the population is overweight (BMI $\geqslant 25$ to $<30 \mathrm{~kg} \cdot \mathrm{m}^{-2}$ ). The obesity epidemic has impacted both developed and developing nations throughout the world. The World Health Organization estimates that worldwide, $\sim 2$ billion people are either obese or overweight.

Obesity is a well-established risk factor for type 2 diabetes, hypertension and atherosclerosis. Obesity is also an important risk factor for asthma. In the late 1980s, two large surveys were published that examined the impact of obesity on the prevalence of chronic diseases $[1,2]$. Both reported an increased prevalence of asthma in the obese and/or overweight. These studies received relatively little attention until $\sim 10$ yrs later, when reports began to emerge describing associations between obesity and asthma in adults and children of multiple ethnicities throughout the world [3]. These association studies were limited by their inability to address the direction of causality and were initially interpreted as indicating a more sedentary lifestyle in asthmatics (due to respiratory symptoms during exercise) resulting in more obesity. It was not until publication of the first prospective analysis of the relationship between obesity and asthma in 1999 [4] that obesity really began to be appreciated as a major risk factor for asthma. The authors followed almost 86,000 initially nonasthmatic females over a 4-yr period. They observed that after adjustment for several factors, including exercise, initial BMI correlated strongly with the risk of newonset asthma. Subsequently, several other prospective studies confirmed these observations [3]. A recent meta-analysis of these data indicates that the odds ratio for incident asthma associated with obesity is 1.92 (95\% CI 1.43-2.59) [5]. These prospective studies were important because they suggested the direction of causality: obesity preceded asthma. Nevertheless, concerns lingered. To ascertain asthma, most of the studies used self reports of a doctor's diagnosis (usually a symptombased diagnosis). Was excess shortness of breath in obese individuals upon exertion being misdiagnosed as asthma? Where the obese more frequently diagnosed because they were seeking physicians' care for other comorbidities? A study by AARON et al. [6] in 2008 largely dispelled concerns related to diagnosis. The authors measured bronchodilator responses and/or airway hyperresponsiveness in >500 randomly selected

Dept of Environmental Health, Harvard School of Public Health, Boston, MA, USA.

CORRESPONDENCE: S.A. Shore, Dept of Environmental Health, Harvard School of Public Health, 665 Huntington Ave, Boston, MA 02115, USA. E-mail: sshore@hsph.harvard.edu subjects self-reporting asthma and could find no difference in the prevalence of asthma misdiagnosis between obese and lean subjects using these objective measures. Other data also supported the likelihood that the relationship between obesity and asthma was real. Studies showed that several different types of obese mice exhibited innate airway hyperresponsiveness and increased responses to certain asthma triggers [7]. Obese asthmatics who lost weight had fewer asthma symptoms and improved lung function, including less airway hyperresponsiveness $[8,9]$.

There are concerns with the use of BMI as an index of adiposity. BMI underestimates the prevalence of obesity, especially in males in whom BMI actually correlates better with lean mass than with body fat percentage [10]. This may explain why many, but not all, of the large epidemiological studies examining the impact of obesity on asthma reported either effects in females but not males or greater odds ratios in female versus male subjects. The imperfection of BMI as a measure of obesity likely has important implications for other studies relating obesity to lung disease, since fat mass and fatfree mass have opposing effects on pulmonary function [11].

Emerging data indicate that the location of body fat is an important determinant of the risk of obesity for asthma, just as it is for other obesity-related conditions [12]. Several association studies indicate that abdominal obesity (waist circumference $>88 \mathrm{~cm}$ in females or $>102 \mathrm{~cm}$ in males) results in greater asthma risk than general obesity (BMI $\geqslant 30 \mathrm{~kg} \cdot \mathrm{m}^{-2}$ ). In one of the largest of these studies, which used a cohort of $>88,000$ female teachers from California, VON BEHREN et al. [13] reported that abdominal obesity was a risk factor for asthma even in females with a normal BMI. Furthermore, among those with general obesity, those who had abdominal obesity had a greater odds ratio for asthma than those with a normal waist size.

In this issue of the European Respiratory Journal, BRUMPTON et al. [14] report the results of the first prospective study examining the impact of abdominal obesity on asthma in both males and females. The authors studied $>23,000$ subjects from the Nord-Trøndelag region of Norway over an 11-yr period between 1995 and 2008. They observed that in both males and females, both general and abdominal obesity were risk factors for new-onset asthma. Moreover, in females, abdominal obesity was a risk factor for asthma even after correcting for general obesity.

The study has numerous strengths. The size and duration of the study, and the racial and environmental uniformity of the study population allowed for sufficient power that, despite strong correlations between general and abdominal obesity, an independent effect of abdominal obesity still emerged. Norway 
has a universal-coverage national healthcare system, reducing confounding that might result from effects of healthcare access on diagnosis. The authors limited their population to those $<65$ yrs of age to reduce the possibility that subjects with chronic obstructive pulmonary disease might be misclassified as asthmatics. The authors also performed a sensitivity analysis on a subgroup, using a stricter definition of asthma that included use of bronchodilators and adjusting the data for a number of covariates including age, physical activity and smoking, and found essentially similar results.

Several hypotheses have been proposed to account for the impact of obesity on asthma [15]. For example, it has been postulated that obesity-related reductions in functional residual capacity (FRC) promote asthma because they reduce airway size and increase airway responsiveness. The observations of BRUMPTON et al. [14] are consistent with this hypothesis, since the ability of obesity to reduce the FRC also depends on the locus of fat distribution. For example, SUTHERLAND et al. [16] noted an inverse correlation between FRC and measures of abdominal obesity, whereas there was no correlation with BMI. Obesity-related reductions in forced expiratory volume in $1 \mathrm{~s}$ are also related to waist circumference but not BMI [17]. In other words, only adipose tissue that impinges on the volume of the chest cavity, and not adipose tissue in the buttocks and hips, appears to affect lung volumes.

Obesity also leads to a state of low-grade systemic inflammation consequent to adipose tissue inflammation, and it has also been postulated that obesity may promote asthma through effects of these elevated concentrations of cytokines, chemokines, adiposederived hormones and acute phase proteins acting on the lung [15]. The observations of BRUMPTON et al. [14] that abdominal obesity has an independent effect on asthma over and above general obesity is also consistent with a role for systemic inflammation in this relationship. In the Framingham Heart Study, the amounts of both abdominal subcutaneous adipose tissue and visceral adipose tissue were associated with markers of systemic inflammation [18]. Moreover, it is conceivable that reductions in lung volume and systemic inflammation are related. Current concepts indicate the adipose tissue inflammation results from local tissue hypoxia leading to adipocyte cell death. Such hypoxia-mediated cell death would be expected to be amplified in the setting of hypoxaemia, and obesity-related hypoxaemia is associated with reductions in lung volume [19] and abdominal obesity [20].

The implications of the observations of BRUMPTON et al. [14] are enormous. In the USA, abdominal obesity is even more prevalent than general obesity: in a 2003-2004 survey, $61 \%$ of the adult female population had abdominal obesity [12]. In addition, the prevalence of abdominal obesity increased with age, reaching almost $75 \%$ in females between the ages of 60 and $69 \mathrm{yrs}$. In the last few years, cluster analyses have reported a group of asthmatics in which obesity is associated with female sex, adult onset of disease, low sputum eosinophils and increased symptoms [21]. Given the very high prevalence of abdominal obesity in females, especially as they age [12], and the increased risk of asthma consequent to abdominal obesity in females [14], it is perhaps not surprising that this phenotype has emerged as the obesity epidemic has progressed.

\section{STATEMENT OF INTEREST}

None declared.

\section{REFERENCES}

1 Seidell JC, de Groot LC, van Sonsbeek JL, et al. Associations of moderate and severe overweight with self-reported illness and medical care in Dutch adults. Am J Public Health 1986; 76: 264-269.

2 Negri E, Pagano R, Decarli A, et al. Body weight and the prevalence of chronic diseases. I Epidemiol Community Health 1988; 42: 24-29.

3 Shore SA, Johnston RA. Obesity and asthma. Pharmacol Ther 2006; 110: 83-102.

4 Camargo CA Jr, Weiss ST, Zhang S, et al. Prospective study of body mass index, weight change, and risk of adult-onset asthma in women. Arch Intern Med 1999; 159: 2582-2588.

5 Beuther DA, Sutherland ER. Overweight, obesity, and incident asthma: a meta-analysis of prospective epidemiologic studies. Am J Respir Crit Care Med 2007; 175: 661-666.

6 Aaron SD, Vandemheen KL, Boulet LP, et al. Overdiagnosis of asthma in obese and nonobese adults. CMAJ 2008; 179: 1121-1131.

7 Shore SA. Obesity and asthma: lessons from animal models. J Appl Physiol 2007; 102: 516-528.

8 Dixon AE, Pratley RE, Forgione PM, et al. Effects of obesity and bariatric surgery on airway hyperresponsiveness, asthma control, and inflammation. J Allergy Clin Immunol 2011; 128: 508-515.

9 Boulet LP, Turcotte H, Martin J, et al. Effect of bariatric surgery on airway response and lung function in obese subjects with asthma. Respir Med 2012; 106: 651-660.

10 Romero-Corral A, Somers VK, Sierra-Johnson J, et al. Accuracy of body mass index in diagnosing obesity in the adult general population. Int J Obesity 2008; 32: 959-966.

11 Lazarus R, Colditz G, Berkey CS, et al. Effects of body fat on ventilatory function in children and adolescents: cross-sectional findings from a random population sample of school children. Pediatr Pulmonol 1997; 24: 187-194.

$12 \mathrm{Li} \mathrm{C}$, Ford ES, McGuire LC, et al. Increasing trends in waist circumference and abdominal obesity among US adults. Obesity (Silver Spring) 2007; 15: 216-224.

13 Von Behren J, Lipsett M, Horn-Ross PL, et al. Obesity, waist size and prevalence of current asthma in the California Teachers Study cohort. Thorax 2009; 64: 889-893.

14 Brumpton B, Langhammer A, Romundstad P, et al. General and abdominal obesity and incident asthma in adults: the HUNT study. Eur Respir J 2013; 41: 323-329.

15 Shore SA. Obesity and asthma: possible mechanisms. J Allergy Clin Immunol 2008; 121: 1087-1093.

16 Sutherland TJ, Goulding A, Grant AM, et al. The effect of adiposity measured by dual-energy $\mathrm{X}$-ray absorptiometry on lung function. Eur Respir J 2008; 32: 85-91.

17 Leone N, Courbon D, Thomas F, et al. Lung function impairment and metabolic syndrome: the critical role of abdominal obesity. Am J Respir Crit Care Med 2009; 179: 509-516.

18 Pou KM, Massaro JM, Hoffmann U, et al. Visceral and subcutaneous adipose tissue volumes are cross-sectionally related to markers of inflammation and oxidative stress: the Framingham Heart Study. Circulation 2007; 116: 1234-1241.

19 Pelosi P, Croci M, Ravagnan I, et al. The effects of body mass on lung volumes, respiratory mechanics, and gas exchange during general anesthesia. Anesth Analg 1998; 87: 654-660.

20 Zavorsky GS, Murias JM, Kim do J, et al. Waist-to-hip ratio is associated with pulmonary gas exchange in the morbidly obese. Chest 2007; 131: 362-367.

21 Haldar P, Pavord ID, Shaw DE, et al. Cluster analysis and clinical asthma phenotypes. Am J Respir Crit Care Med 2008; 178: 218-224. 